\title{
Efficient Detection of FSK-signals Based on Cyclic Statistics
}

\author{
Yi Zhou, Khalid Qaraqe and Erchin Serpedin \\ Department of Electrical \& Computer Engineering \\ Texas A\&M University \\ College Station, TX 77843-3128 \\ Email: serpedin@ece.tamu.edu
}

\author{
Octavia Dobre \\ Faculty of Engineering and Applied Science \\ Memorial University of Newfoundland \\ St. John's, NL A1B 3X5, Canada \\ Email: dobre@engr.mun.ca
}

\begin{abstract}
Cognitive radios represent a powerful technology for improving the utilization of radio spectrum resources. Detection of low signal-to-noise ratio (SNR) signals with relaxed a priori information on the signal parameters is of high importance for cognitive radios. This paper proposes a detection algorithm based on the first-order cyclostationarity (or cyclic statistics) for frequency shift keying (FSK) signals that only requires partial knowledge on the signal bandwidth, carrier frequency and symbol rate. The results of both theoretical asymptotic analysis and computer simulations are presented. It is shown that the proposed algorithm performs well at low SNRs.
\end{abstract}

Index Terms-Cognitive Radio, Cyclostationarity, Signal Detection, Frequency Shift Keying

\section{INTRODUCTION}

The need for higher data rates is increasing as a result of the transition from conventional wireless voice-oriented communications to wireless multimedia applications. Given the scarcity of the usable frequency spectrum, it turns out that static frequency allocation schemes cannot accommodate the need for increased data rates. Therefore, new ways for exploiting the available spectrum are needed. Cognitive radios represent intelligent wireless communication systems capable of sensing and adapting their features to the existing radio frequency environment [1]-[3]. The most important component of cognitive radios is their ability to measure, sense and be aware of the radio channel characteristics, detect availability of spectrum and received signal features (power, modulation) to opportunistically search for and exploit the unoccupied spectrum [4]-[7]. Since a significant portion of the spectrum allocated to licensed services is sparsely occupied at any given time, a dynamic spectrum allocation strategy can yield major benefits. Notice also that intelligent radios are also required for real-time signal interception and processing in many other applications. However, a major issue in cognitive radios is the detection of signals of low signal-to-noise power ratio (SNR) assuming as little as possible a priori information about the signal parameters.

In this paper, the first-order cyclostationarity (cyclic statistics) of band-limited frequency shift keying (FSK) signals that are affected by additive Gaussian noise, carrier phase and frequency offset, and time delay is investigated for detection. A first-order cyclic moment based detector is proposed, which requires only rough information about the signal bandwidth, carrier frequency and symbol rate. Theoretical analysis for the asymptotic performance of the first-order cyclic moment based detector is conducted. The rest of the paper is organized as follows. The signal model and the first-order cyclostationarity are introduced in Section II. The asymptotic analysis for the cyclostationary statistics of the signals of interest is performed in Section III. Section IV proposes a detection algorithm based on the first-order cyclic moments of FSK signal and its asymptotic (large sample) performance analysis is carried out therein. Section V concludes the paper.

\section{First-Order CyClostationARity of Amplitude MODUlated SigNALS}

\section{A. Signal Model}

Assuming rough information on the signal bandwidth and carrier frequency, the out-of-band noise and interferences are removed by appropriate filtering and the received signal is then down-converted yielding the signal model:

$$
r(t)=x(t)+\nu(t)
$$

where $x(t)$ represents the transmitted signal, which is affected by phase, frequency offset and time delay, and $\nu(t)$ denotes the additive zero-mean Gaussian noise. For an M-FSK signal, $x(t)$ is expressed as

$$
x(t)=A e^{j \theta} e^{j 2 \pi \Delta f t} \sum_{i} e^{j 2 \pi f_{d} s_{i}\left(t-i T_{s}-t_{0}\right)} g\left(t-i T_{s}-t_{0}\right),
$$

where $A, \theta$ and $\Delta f$ stand for the signal amplitude, carrier phase and frequency offset, respectively. The variable $f_{d}$ stands for the frequency deviation, $T_{s}$ denotes the symbol period, $t_{0}$ represents the time delay, $g(t)$ stands for the signal pulse shape, and $s_{i}$ is the symbol transmitted within $i$ th period. The data symbols $\left\{s_{i}\right\}$ are assumed to be zeromean independent and identically distributed (i.i.d.) random variables, with values drawn from the alphabet $\mathcal{A}_{\mathrm{M}-\mathrm{FSK}}=$ $\left\{s_{m}: s_{m}=2 m-1-M, m=1, \cdots, M\right\}$, with $M$ denoting the modulation order.

\section{B. First-Order Cyclostationary Statistics}

The received signal is sampled at the output of mixer with the sampling rate $f_{s}=1 / T_{s}$ yielding the discrete-time first- 
order cyclostationary signal

$$
r[n]=\left.r(t)\right|_{t=n T_{s}} .
$$

The signal $r[n]$ is a discrete-time first-order cyclostationary process, and its first-order time-varying moment $\mu_{r}[n]=$ $\mathbf{E}\{r[n]\}$ is an almost periodic function of time which admits the Fourier series expansion [8]

$$
\mu_{r}[n]=\sum_{\alpha \in \kappa} m_{r}(\alpha) e^{j 2 \pi \alpha n},
$$

where $\kappa=\left\{\alpha: m_{r}(\alpha) \neq 0\right\}$ represents the set of first-order cyclic frequencies (CFs) and $m_{r}(\alpha)$ denotes the first-order cyclic moment (CM) at $\mathrm{CF} \alpha$, and is defined as follows

$$
m_{r}(\alpha)=\lim _{N \rightarrow \infty} \frac{1}{N} \sum_{n=0}^{N-1} \mu_{r}[n] e^{-j 2 \pi \alpha n} .
$$

The estimator of the first-order CM at CF $\alpha$ based on $N$ samples is given by [9]

$$
\hat{m}_{r}^{(N)}(\alpha)=\frac{1}{N} \sum_{n=0}^{N-1} r[n] e^{-j 2 \pi \alpha n} .
$$

\section{ASYMPTOTIC ANALYSIS}

In this section, the asymptotic statistic of the first-order CM in the presence of a large observation size is presented. Based on the asymptotic analysis, we propose a detection algorithm for FSK signals in the next section.

\section{A. Asymptotic Statistics for FSK Signals}

When an FSK signal is present at the receiver, assuming prior knowledge of symbol duration is available and ignoring the noise, the sampled received signal can be expressed as

$$
\begin{aligned}
r[n] & =A e^{j \theta} e^{j 2 \pi \Delta f T_{s} n} \sum_{i=-\infty}^{\infty} e^{j 2 \pi f_{d} s_{i}\left(n T_{s}-i T_{s}-t_{0}\right)} g[n-i] \\
& =A e^{j \theta} e^{j 2 \pi \Delta f T_{s} n} \sum_{i=-\infty}^{\infty} e^{-j 2 \pi f_{1} s_{i}} e^{j 2 \pi f_{2} s_{i}(n-i)} g[n-i],
\end{aligned}
$$

where $g[n]=g\left(n T_{s}-t_{0}\right), f_{1}=f_{d} t_{0}$, and $f_{2}=f_{d} T_{s}$. It follows that

$$
\begin{aligned}
& \mathbf{E}\left\{\hat{m}_{r}^{(N)}(\alpha)\right\}=\frac{1}{N} \sum_{n=0}^{N-1} \mathbf{E}\{r[n]\} e^{-j 2 \pi \alpha n} \\
= & \frac{A e^{j \theta}}{M N} \sum_{m=1}^{M} e^{-j 2 \pi f_{1} s_{m}} \sum_{i=-\infty}^{\infty} \sum_{n=0}^{N-1} e^{j 2 \pi f_{2} s_{m}(n-i)} g[n-i] e^{j 2 \pi f_{0} n} \\
= & \frac{A e^{j \theta}}{M N} \sum_{m=1}^{M} e^{-j 2 \pi f_{1} s_{m}} \sum_{\tau=-\infty}^{\infty} g[\tau] e^{j 2 \pi f_{2} s_{m} \tau} \sum_{i=-\tau}^{N-1-\tau} e^{j 2 \pi f_{\bullet}(i+\tau)} \\
= & \frac{A e^{j \theta} e^{j \pi f_{0}(N-1)}}{M} \sum_{m=1}^{M} e^{-j 2 \pi f_{1} s_{m}} G^{*}\left(e^{j 2 \pi f_{2} s_{m}}\right) \frac{\sin \left(\pi f_{0} N\right)}{N \sin \left(\pi f_{0}\right)}
\end{aligned}
$$

where $G\left(e^{j 2 \pi f}\right)=\sum_{n=-\infty}^{\infty} g[n] e^{-j 2 \pi f n}, f_{0}=\Delta f T_{s}-\alpha$. For ideal brick wall low-pass filtering at the frond end of the receiver, and with Nyquist sampling, $G\left(e^{j 2 \pi f}\right)=1, \forall f$. Hence, for any given CF $\alpha=\Delta f T_{s}-f_{0}$,

$$
\mathbf{E}\left\{\hat{m}_{r}^{(N)}(\alpha)\right\}=A e^{j \theta} e^{j \pi f_{0}(N-1)} \frac{\sin \left(2 \pi f_{1} M\right) \sin \left(\pi f_{0} N\right)}{M \sin \left(2 \pi f_{1}\right) N \sin \left(\pi f_{0}\right)} .
$$

It is known that

$$
\operatorname{var}\left\{\hat{m}_{r}^{(N)}(\alpha)\right\}=\mathbf{E}\left\{\left|\hat{m}_{r}^{(N)}(\alpha)\right|^{2}\right\}-\left|\mathbf{E}\left\{\hat{m}_{r}^{(N)}(\alpha)\right\}\right|^{2}
$$

After some tedious manipulations, it follows that

$$
\begin{aligned}
\lim _{N \rightarrow \infty} N \operatorname{var}\left\{\hat{m}_{r}^{(N)}(\alpha)\right\}= & A^{2}\left\{1+\left[2 \pi \sum_{k=-\infty}^{\infty} \delta\left(f_{0}-k\right)-1\right]\right. \\
& \left.\times\left[\frac{\sin \left(2 \pi f_{1} M\right)}{M \sin \left(2 \pi f_{1}\right)}\right]^{2}\right\} .
\end{aligned}
$$

It follows that when $f_{0}=\Delta f T_{s}-\alpha \in \mathbf{Z}$ is an integer,

$\lim _{N \rightarrow \infty} N \operatorname{var}\left\{\hat{m}_{r}^{(N)}(\alpha)\right\}=A^{2}\left\{1+(2 \pi-1)\left[\frac{\sin \left(2 \pi f_{1} M\right)}{M \sin \left(2 \pi f_{1}\right)}\right]^{2}\right\}$,

whereas for an non-integer frequency $f_{0}=\Delta f T_{s}-\alpha \notin \mathbf{Z}$,

$$
\lim _{N \rightarrow \infty} N \operatorname{var}\left\{\hat{m}_{r}^{(N)}(\alpha)\right\}=A^{2}\left\{1-\left[\frac{\sin \left(2 \pi f_{1} M\right)}{M \sin \left(2 \pi f_{1}\right)}\right]^{2}\right\} .
$$

It can be shown that, for large enough sample size $N$, $\hat{m}_{r}^{(N)}(\alpha)$ asymptotically follows a complex normal distribution $\mathcal{C N}\left(A e^{j \theta} e^{j \pi f_{0}(N-1)} \frac{\sin \left(2 \pi f_{1} M\right)}{M \sin \left(2 \pi f_{1}\right)} \frac{\sin \left(\pi f_{0} N\right)}{N \sin \left(\pi f_{\bullet}\right)}, A^{2} / N(1-\right.$ $\left.\left.\left[\frac{\sin \left(2 \pi f_{1} M\right)}{M \sin \left(2 \pi f_{1}\right)}\right]^{2}\right)\right)$ at $\mathrm{CF} \alpha \neq \Delta f T_{s}+k, k \in \mathbf{Z}$.

\section{B. Cyclostationary Statistics of Noise}

The noise $\nu[n]$ is the output of the receive low-pass filter, and assumes the following expression

$$
\nu[n]=\sum_{i=-\infty}^{\infty} w[n-i] g[i]
$$

where $\{w[n]\}$ are i.i.d. complex Gaussian random variables with zero mean and variance $\sigma^{2}$. It follows that

$$
\begin{aligned}
\mathbf{E}\left\{\nu[n] \nu^{*}[m]\right\} & =\mathbf{E}\left\{\sum_{i=-\infty}^{\infty} w[n-i] g[i] \sum_{k=-\infty}^{\infty} w^{*}[m-k] g[k]\right\} \\
& =\sum_{i=-\infty}^{\infty} \sum_{k=-\infty}^{\infty} \mathbf{E}\left\{w[n-i] w^{*}[m-k]\right\} g[i] g[k] \\
& =\sigma^{2} \sum_{k=-\infty}^{\infty} g[k] g[n-m+k]
\end{aligned}
$$

and

$$
\mathbf{E}\left\{\hat{m}_{\nu}^{(N)}(\alpha)\right\}=\frac{1}{N} \sum_{n=0}^{N-1} \mathbf{E}\{\nu[n]\} e^{-j 2 \pi \alpha n}=0
$$


The variance of the first-order cyclostationary moment estimator is given by

$$
\begin{aligned}
\operatorname{var}\left\{\hat{m}_{r}^{(N)}(\alpha)\right\} & =\mathbf{E}\left\{\left|\hat{m}_{\nu}^{(N)}(\alpha)\right|^{2}\right\} \\
& =\frac{1}{N^{2}} \sum_{n=0}^{N-1 N-1} \sum_{m=0}^{N} \mathbf{E}\left\{\nu[n] \nu^{*}[m]\right\} e^{-j 2 \pi \alpha(n-m)} \text {. (5) }
\end{aligned}
$$

Substituting Eq. (4) into Eq. (5), it follows that

$$
\begin{aligned}
& \lim _{N \rightarrow \infty} N \operatorname{var}\left\{\hat{m}_{r}^{(N)}(\alpha)\right\} \\
= & \lim _{N \rightarrow \infty} \frac{\sigma^{2}}{N} \sum_{n=0}^{N-1} \sum_{m=0}^{N-1} \sum_{k=-\infty}^{\infty} g[k] g[n-m+k] e^{-j 2 \pi \alpha(n-m)} \\
= & \lim _{N \rightarrow \infty} \sigma^{2} \sum_{\tau=-(N-1)}^{N-1}\left(1-\frac{|\tau|}{N}\right) \sum_{k=-\infty}^{\infty} g[k] g[k+\tau] e^{-j 2 \pi \alpha \tau} \\
= & \sigma^{2}\left|G\left(e^{j 2 \pi \alpha}\right)\right|^{2}
\end{aligned}
$$

where the last equality follows the convergence of Cesàro sum [10] and $G\left(e^{j 2 \pi \alpha}\right)=\sum_{n=-\infty}^{\infty} g[n] e^{-j 2 \pi \alpha n}$ stands for the discrete-time Fourier transform of $g[n]$.

Thus, for a large enough sample size $N$, the firstorder $\mathrm{CM}$ at $\mathrm{CF} \alpha$ with noise only can be modeled as a complex Gaussian random variable with the distribution $\mathcal{C N}\left(0, \sigma^{2}\left|G\left(e^{j 2 \pi \alpha}\right)\right|^{2} / N\right)$.

\section{Detection Algorithm and Performance ANALYSIS}

This section proposes first an algorithm for detection of FSK signals by exploiting the first-order cyclostationary statistics. Then a theoretical performance analysis is carried out. The detection of the FSK signal is formulated as a hypothesis testing problem. The received signal is FSK if first-order CF components are detected. Otherwise, there is no signal (only noise) is presented at the receiver. The detection algorithm consists of the following two steps.

Step I: Based on Eq. (1), the first-order CM of the received signal is estimated based on a $N$-sample observation interval, at candidate CFs $\alpha^{\prime}$ over the range corresponding to the signal bandwidth normalized to the sampling rate. The first-order CM for FSK signals are non-zero at most CFs, whereas for noise the $\mathrm{CM}$ is statistically insignificant and close to zero. For FSK signals, the magnitude of the first-order CM peaks at the CFs $\alpha=\Delta f T_{s} \pm k$, where $k=0, \pm 1, \cdots$ is an integer.

Step II: The magnitude of the first-order CM is compared against a pre-chosen threshold $T$. If $\left|\hat{m}_{r}^{(N)}\left(\alpha^{\prime}\right)\right|>T$, there is signal of interest present, otherwise not. The threshold $T$ is determined according to a given false alarm rate. For FSK signals, there are multiple peaks present.

For large enough $N$, when the FSK signal is present, the magnitude of $\mathrm{CM}$ at $\mathrm{CF} \alpha=\Delta f T_{s}$ follows the Rician distribution with probability density function (pdf) $f_{2}(x)$

$$
\left|\hat{m}_{r}^{(N)}(\alpha)\right| \sim f_{2}(x)=\left\{\begin{array}{ll}
\frac{x}{\sigma_{2}^{2}} e^{-\frac{x^{2}+B^{2}}{2 \sigma_{2}^{2}}} I_{0}\left(\frac{B x}{\sigma_{2}^{2}}\right) & x \geq 0 \\
0 & x<0
\end{array},\right.
$$

where $I_{0}(x)$ stands for the zeroth order modified Bessel function of the first kind, and $B=A \frac{\sin \left(2 \pi f_{1} M\right)}{M \sin \left(2 \pi f_{1}\right)} \frac{\sin \left(\pi f_{0} N\right)}{N \sin \left(\pi f_{0}\right)}, \sigma_{2}^{2}=$ $A^{2} / 2 N\left(1-\left[\frac{\sin \left(2 \pi f_{1} M\right)}{M \sin \left(2 \pi f_{1}\right)}\right]^{2}\right)+\sigma^{2}\left|G\left(e^{j 2 \pi \Delta f T_{s}}\right)\right|^{2} / 2 N$.

When there is noise only, the magnitude of CM at CF $\alpha=$ $\Delta f T_{s}$ follows the Rayleigh distribution

$$
\left|\hat{m}_{r}^{(N)}(\alpha)\right| \sim f_{3}(x)=\left\{\begin{array}{ll}
\frac{x}{\sigma_{3}^{2}} e^{-\frac{x^{2}}{2 \sigma_{3}^{2}}} & x \geq 0 \\
0 & x<0
\end{array},\right.
$$

where $\sigma_{3}^{2}=\sigma^{2}\left|G\left(e^{j 2 \pi \Delta f T_{s}}\right)\right|^{2} / 2 N$.

We adopt a detector with threshold $T\left(P_{F}\right)$ such that a constant false alarm rate $P_{F}$ is achieved. The false alarm rate is given by

$$
P_{F}=\int_{T}^{\infty} f_{3}(x) d x
$$

whereas the detection probability is

$$
P_{D}=\int_{T}^{\infty} f_{2}(x) d x
$$

The performance of the detector can be evaluated only numerically. Note that the right-tail probability of the Rician random variable can be related to that of the noncentral chi-square random variable as depicted before [10] by the following equations:

$$
\operatorname{Pr}\{x>\gamma\}=Q_{\chi_{2}^{\prime 2}(\lambda)}\left(\frac{\gamma^{2}}{\sigma_{2}^{2}}\right),
$$

where $\lambda=A^{2} / \sigma_{2}^{2}$, and for $x>0$

$$
\begin{aligned}
Q_{\chi_{\nu}^{\prime 2}(\lambda)}(x) & =\int_{x}^{\infty} \frac{x^{\frac{\nu}{2}-1} \exp \left[-\frac{1}{2}(x+\lambda)\right]}{2^{\frac{\nu}{2}}} \sum_{k=0}^{\infty} \frac{\left(\frac{\lambda x}{4}\right)^{k}}{k ! \Gamma\left(\frac{\nu}{2}+k\right)} \\
\Gamma(u) & =\int_{0}^{\infty} t^{u-1} e^{-t} d t
\end{aligned}
$$

Figs. 1 - 2 illustrate the Receiver Operating Characteristics (ROC) of the proposed detection algorithm for FSK signals with constant false alarm rates of 0.05 and 0.01 , and when the sample size $N$ is equal to 10 , and 40 samples, respectively. Ideal low-pass receive filtering and Nyquist sampling are applied and the SNR knowledge is assumed. Binary FSK modulation is adopted.

From Figs $1-2$, one can find that the detection algorithm works well in the SNR region that is typical for cognitive radio applications and is insensitive to the sample size.

\section{CONCLUSIONS}

A detection algorithm based on the first-order cyclostationarity is proposed for FSK signals with relaxed a priori information on signal parameters. Theoretical asymptotic analysis and numerical simulations are carried out to demonstrate the performance of the proposed algorithm. It has been shown that the algorithm performs reasonably well in the SNR range typical for cognitive radio. Future work will be conducted for signals with different modulation formats and more complex and challenging propagation environments. 


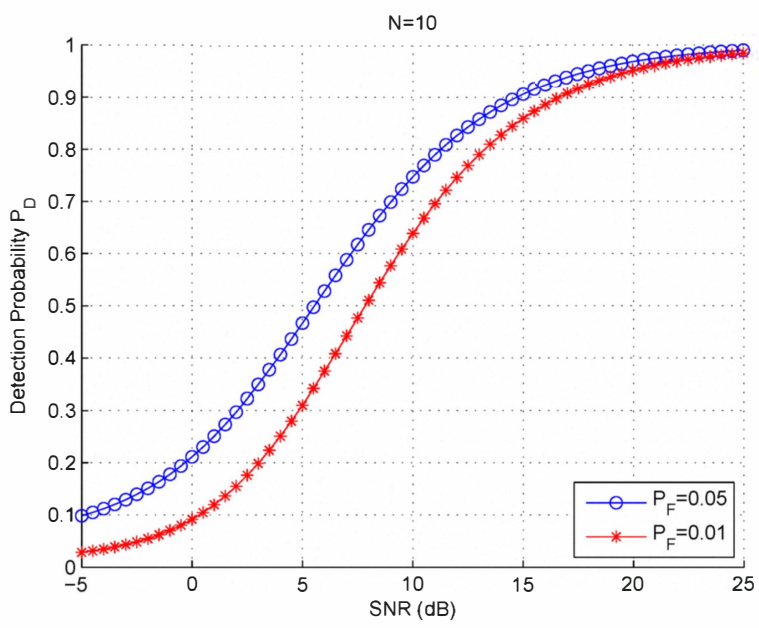

Fig. 1. Detection probabilities with $\mathrm{SNR}, \mathrm{N}=10$.

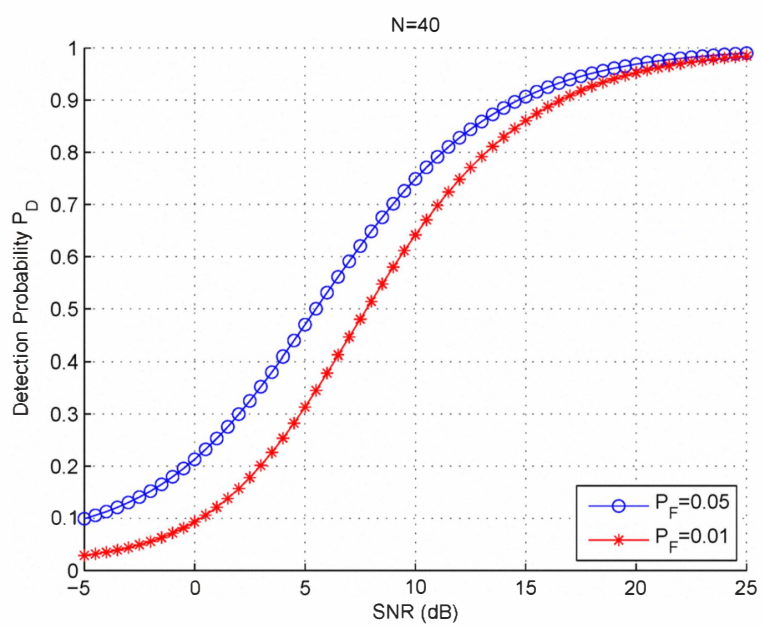

Fig. 2. Detection probabilities with $\mathrm{SNR}, \mathrm{N}=40$.

\section{ACKNOWLEDGMENT}

This work was supported in part by Qtel and QNRF-NPRP.

\section{REFERENCES}

[1] S. Haykin, Cognitive radio: brain-empowered wireless communications, IEEE Journal on Selected Areas in Communications, vol. 23, no. 2, pp 201220, Feb. 2005

[2] H. Celebi and H. Arslan, Adaptive positioning systems for cognitive radios, in 2nd IEEE International Symposium on New Frontiers in Dynamic Spectrum Access Networks, 2007. DySPAN 2007., April 2007, pp. 7884

[3] X. Liu, G. Zhao, and X. Ma, Target localization and tracking in noisy binary sensor networks with known spatial topology, Wireless Communications and Mobile Computing, vol. 9, no. 8, pp. 10281039 , July 2008.

[4] S. Haykin, D. Thomson, and J. Reed, Spectrum sensing for cognitive radio, Proceedings of the IEEE, vol. 97, no. 5, pp. 849877, May 2009

[5] T. Yucek and H. Arslan, A survey of spectrum sensing algorithms for cognitive radio applications, IEEE Communications Surveys and Tutorials, vol. 11, no. 1, pp. 116130, Quarter 2009.

[6] Z. Quan, S. Cui, and A. Sayed, Optimal linear cooperation for spectrum sensing in cognitive radio networks, IEEE Journal of Selected Topics in Signal Processing, vol. 2, no. 1, pp. 2840, Feb. 2008.
[7] Z. Tian and G. Giannakis, Compressed sensing for wideband cognitive radios, in IEEE International Conference on Acoustics, Speech and Signal Processing, 2007. ICASSP 2007., vol. 4, April 2007, pp. 1357 1360 .

[8] W. A. Gardner, Cyclostationarity in Communications and Signal Processing. IEEE Press, 1994.

[9] A. Dandawate and G. Giannakis, Asymptotic theory of mixed time averages and kth-order cyclic-moment and cumulant statistics, IEEE Transactions on Information Theory, vol. 41, no. 1, pp. 216232, Jan 1995.

[10] B. Porat, Digital Processing of Random Signals: Theory and Methods Dover Publications, 2008

[11] S. M. Kay, Fundamentals of Statistical Signal Processing, Volume II Detection Theory. Prentice Hall, 1998 